\title{
N93-26725:
}

\section{A Revised Catalog of CfA Galaxy Groups \\ in the Virgo/Great Attractor Flow Field}

(Ap. J. submittal)

Richard Nolthenius

UCO/Lick Observatory

University of California, Santa Cruz

A new identification of groups and clusters in the CfA1 Catalog of Huchra, et al. (1983) is presented, using a percolation algorithm to identify density enhancements. The procedure differs from that of the original Geller and Huchra (1983; GH) catalog in several important respects; galaxy distances are calculated from the Virgo - Great Attractor flow model of Faber and Burstein (1988), the adopted distance linkage criteria is only $\sim 1 / 4$ as large as in the Geller and Huchra catalog, the sky link relation is taken Erom Nolthenius and white (1987), correction for interstellar extinction is included, and "by-hand" adjustments to group memberships are made in the complex regions of Virgo / Coma I / Ursa Major and Coma / A1367 (to allow for varying group velocity dispersions and to trim unphysical "spider arms"). Since flow model distances are poorly determined in these same regions, available distances from the IR Tully-Fisher, $D_{n}-\sigma$, planetary nebula luminosity function, and surface brightness resolution methods are adopted if possible.

The percolation algorithm's link criteria are cast in terms of a new determination of the galaxy luminosity function $\phi$, using the inhomegeneity-independent procedure of deLapparent, Geller and Huchra (1989). The resulting Schechter parameters are; $\alpha=-1.01$ and $\mathrm{M}^{*}=-19.45$; about 0.2 brighter than earlier determinations $(\alpha$ $=-0.77, \mathrm{M}^{*}=-19.00$ after correction for Malmquist bias). The sky $D_{\mathrm{L}}$ and redshift $\mathrm{V}_{\mathrm{L}}$ link criteria are $\left(\mathrm{V}_{\mathrm{F}} \equiv 5000 \mathrm{~km} \mathrm{~s}^{-1} ; \boldsymbol{\Phi} \equiv\right.$ integrated $\phi$ visible at distance $V)$ :

$$
\begin{gathered}
D_{L}(V) \propto\left[\frac{\Phi(V)}{\Phi\left(V_{p}\right)}\right]^{-1 / 2} L^{-1 / 6}=1.12 \mathrm{MpC}\left[\frac{\Phi(n)}{\Phi\left(V_{F}\right)}\right]^{-1 / 2}\left(\frac{V}{V_{p}}\right)^{-1 / 3} . \\
V_{L}(V)=350 \mathrm{~km} \mathrm{~s}^{-1}\left[\frac{(V)}{\Phi\left(V_{F}\right)}\right]^{-1 / 3}
\end{gathered}
$$

Table 1 compares the global properties of the original GH and new group catalogs. GH's higher mass-to-light and velocity dispersion values and low crossing times are caused by their much higher velocity link criterion. Most groups appear in both catalogs, although $\mathrm{GH}$ groups often contain extra members. The new catalog does better at resolving the complex Virgo / Coma I / Ursa Major / Coma Cluster areas into associated and infalling smaller groups. The group luminosity function is roughly a power law with a possible turn-over at low luminosity. 
Table 1: Group Catalog Global Properties

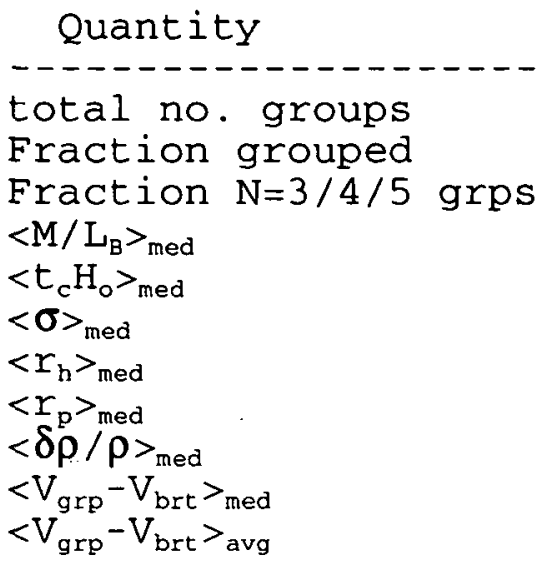

\begin{tabular}{c} 
Value \\
\hline 173 \\
$1146 / 2404=48 \%$ \\
$.40 / .21 / .09$ \\
148 \\
0.444 \\
$116 \mathrm{~km} \mathrm{~s}^{-1}$ \\
$0.48 \mathrm{Mpc}$ \\
$0.72 \mathrm{Mpc}$ \\
119 \\
$6 \mathrm{~km} \mathrm{~s}^{-1}$ \\
$5 \mathrm{~km} \mathrm{~s}^{-1}$
\end{tabular}

GH Value

176

$1451 / 2390=61 \%$

$.33 / .21 / .10$

551

0.271

$231 \mathrm{~km} \mathrm{~s}^{-1}$

$0.55 \mathrm{MpC}$

$---$

$30 \mathrm{~km} \mathrm{~s}^{-1}$

$108 \mathrm{~km} \mathrm{~s}^{-1}$

Contamination by interlopers has been significantly reduced. Figure 1 and Table 1 shows that the GH groups' brightest member tends to be at lower redshift than the group mean, implying comtamination by interlopers in the Hubble flow. The new groups' close $\mathrm{V}_{\mathrm{grp}}-\mathrm{V}_{\mathrm{brt}}$ symmetry about zero implies minimal contamination.

\section{References}

deLapparent, V., Geller, M. J., and Huchra, J. 1989, ApJ 343, 1 Faber, S. M., and Burstein 1988, in Large Scale Motions in the Universe, Proceedings of the Pontifical Academy of Sciences Study Week no. 27 (FB)

Geller, M., and Huchra, J. 1983, ApJS 52, 61 (GH)

Huchra, J., Davis, M., Latham, D., and Tonry, J. 1983, ApJs 52, 89 Nolthenius, R., and white, S. D. M. 1987, MNRAS 225, 505 (NW)

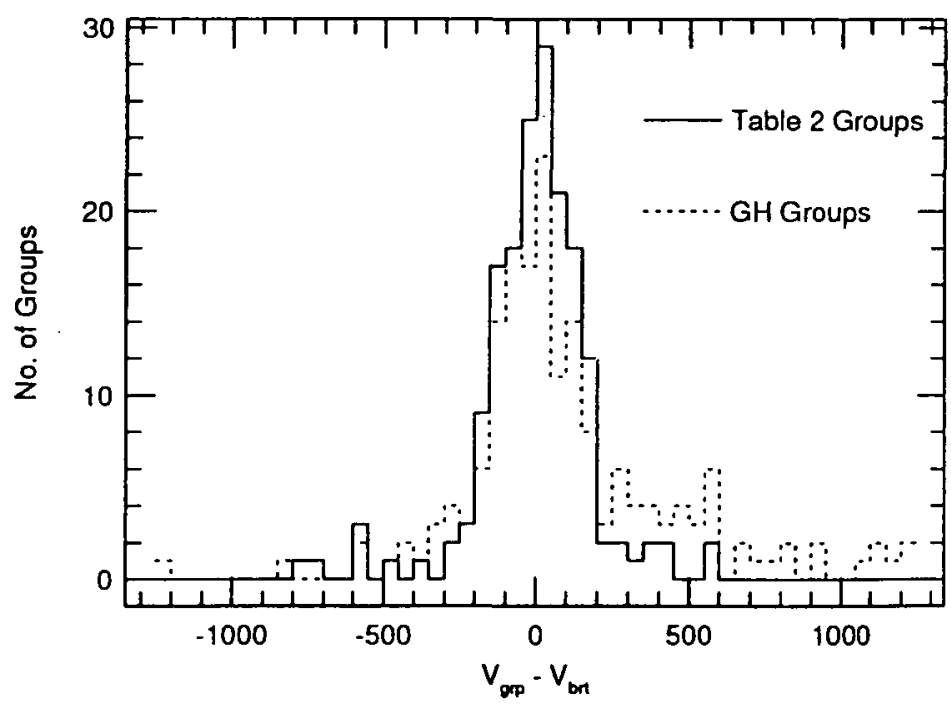

Figure 1. The distribution of residuals $\mathrm{V}_{\mathrm{grp}}-\mathrm{V}_{\mathrm{brt}}$ for the $\mathrm{GH}$ and new groups. The GH catalog's excess of positive $V_{g r p}-V_{b r t}$ groups implies significant interloper contamination compared to the new groups, whose distribution is symmetric about zero. 